02.2;04.1;12.1

\title{
Модель ускорения лазерной плазмы в полях спиральной электродинамической линии
}

\author{
(C) А.Е. Шиканов, Е.Д. Вовченко, К.И. Козловский, В.Л. Шатохин \\ Национальный исследовательский ядерный университет „МИФИ“, \\ Москва, Россия \\ 『E-mail: vlshatokhin@mephi.ru
}

Поступило в Редакцию 19 сентября 2018 г.

\begin{abstract}
Рассматривается ускорение сгустка лазерной плазмы в быстро нарастающем магнитном поле сложной геометрии, для формирования которого применена коническая спираль, расширяющаяся в сторону предполагаемого ускорения плазмы. Приводится алгоритм расчета продольного магнитного поля в приближении замены спиральной линии системой из колец переменного радиуса. Для анализа эффективности ускорения предложена математическая модель, основанная на взаимодействии магнитного момента плазменного сгустка с градиентом спада продольного магнитного поля. Показана возможность достижения дейтронами средней скорости, превышающей $10^{6} \mathrm{~m} / \mathrm{s}$.
\end{abstract}

DOI: 10.21883/PJTF.2019.03.47277.17530

В работе [1] приведены результаты исследования ускорения лазерной плазмы (ЛП), содержащей дейтроны, в нестационарном неоднородном магнитном поле, спадающем в продольном направлении. Рассмотрен случай, когда такое поле создается тонким кольцом радиуса $\sim 0.01 \mathrm{~m} \mathrm{c}$ током, генерируемым при разряде на него емкостного накопителя энергии. Эксперименты показали, что в такой системе ускорения можно получать потоки дейтронов с максимальной энергией в спектре, превышающей $50 \mathrm{keV}$. Это свидетельствует о принципиальной возможности применения подобного рода ускорителей в качестве портативных импульсных генераторов нейтронов (ИГН) на основе ядерных реакций $D(d, n)^{3} \mathrm{He}$ и $T(d, n)^{4} \mathrm{He}$, в которых в отличие от ИГН на основе методов прямого ускорения отсутствуют ограничения на нейтронный выход, связанные с влиянием пространственного заряда на плотность тока дейтронов и эффективность их ускорения [2].

В настоящей работе для повышения эффективности ускорения исследуется возможность применения более сложной пространственно-временно́й структуры магнитного поля, формируемой с помощью электродинамической линии в виде спирали, расширяющейся в сторону предполагаемого ускорения ЛП (рис. 1).

Так же как и в ускорителе с кольцевым контуром с током, в рассматриваемом случае используется индукционный механизм ускорения, основанный на взаимодействии внешнего переменного магнитного поля с ЛП, являющейся проводящей средой. Плазма создается при воздействии интенсивного излучения импульсного лазера на твердотельную мишень из дейтерированного полиэтилена. Лазерная мишень охватывается индукционной катушкой (электродинамической спиральной линией), в которой возбуждается импульс тока, синхронизированный с импульсом лазера.

Быстро нарастающее магнитное поле индуцирует в сгустке лазерной плазмы вихревой ток. Азимутальная составляющая этого тока взаимодействует с радиальной составляющей магнитного поля и создает пондеромоторную силу Ампера, ускоряющую плазму в аксиальном направлении. Ее продольная составляющая, действующая на единичный объем ЛП, определяется выражением $f_{z}(r, z, t)=j(r, z, t) B_{r}(r, z, t)$, где $j(r, z, t)$ и $B_{r}(r, z, t)$ - соответственно плотность азимутального электрического тока и радиальная составляющая магнитного поля в точке с цилиндрическими координатами $r, z$ в плазменном объеме в момент времени $t$.

Следует отметить, что в рамках предложенной модели ускорения ЛП рассматривается как сплошная среда, в которой на макроскопическом уровне выполняются условия квазинейтральности. Это снимает ограничения, связанные с пространственным зарядом. Ионы ускоряются в присутствии электронов. При этом основная энергия ускоренной ЛП из-за разности масс определяется ионами.

Для изучения возможностей эффективного применения спиральной линии при формировании магнитного поля был разработан алгоритм расчета полей, при реализации которого магнитное поле спирали приближенно представлялось в виде суперпозиции полей, создаваемых системой из $N$ колец с током $I(t)$, расположенных в параллельных плоскостях, отстоящих друг от друга на расстояние $H /(N-1)$, где $H-$ продольная длина спирали. Продольные координаты колец определялись формулой $z_{i}(N)=H(i-1) /(N-1)$, где индекс $i=1-N$ означает номер кольца, а радиусы колец возрастают с номером кольца в соответствии с формулой $a_{i}(N)=R_{\min }+\left(R_{\max }-R_{\min }\right)(i-1) /(N-1)$. Для компьютерного моделирования магнитного поля были выбраны геометрические параметры спирали, планируемые в эксперименте на лабораторной установке:

$$
R_{\text {min }}=10^{-2} \mathrm{~m}, \quad R_{\max }=5 \cdot 10^{-2} \mathrm{~m}, \quad H=2.5 \cdot 10^{-2} \mathrm{~m} .
$$




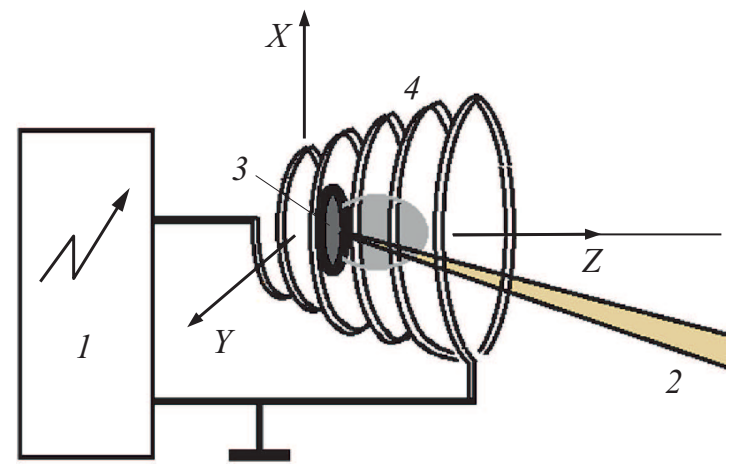

Рис. 1. Схема ускорителя лазерной плазмы с электродинамической спиральной линией. 1 - генератор импульсного напряжения, 2 - лазерный луч, 3 - лазерная мишень, 4 спиральная линия.

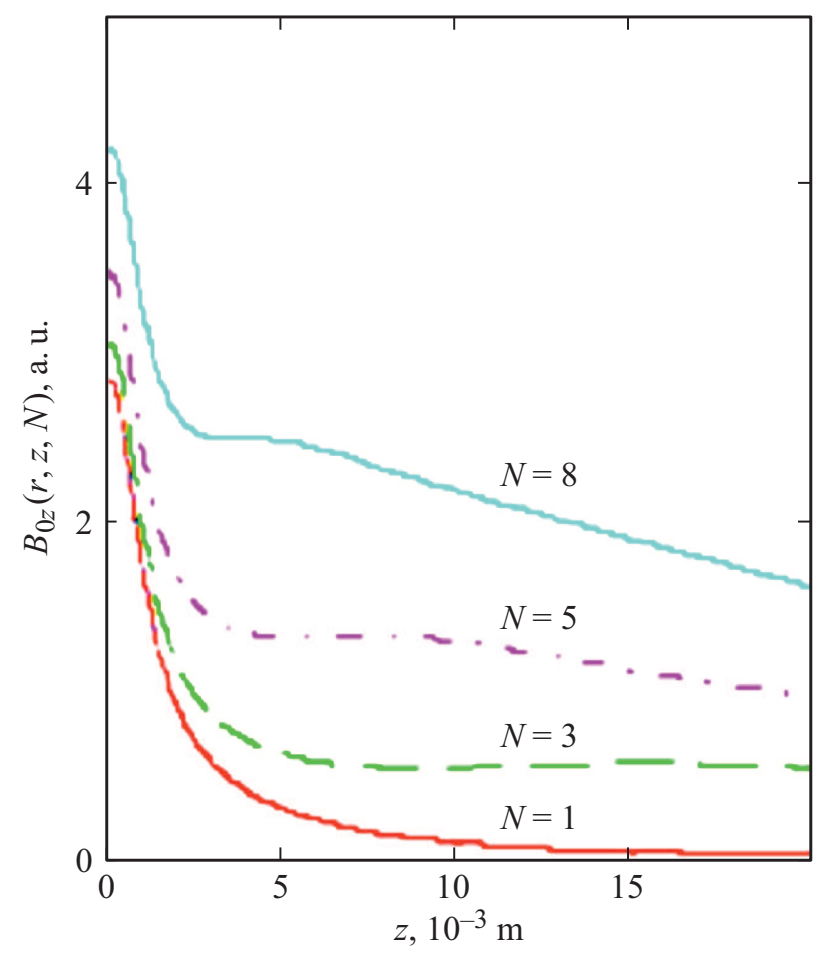

Рис. 2. Зависимости продольного магнитного поля $B_{0 z}$ от координаты $z$ при отклонении от оси на величину $r=4 \cdot 10^{-3} \mathrm{~m}$.

Продольный компонент вектора индукции определялся следующим образом:

$$
\begin{aligned}
B_{z}(r, z, t, N)= & I(t) B_{z}(r, z, N) \\
& =I(t) \sum_{i=1}^{N} b_{z}\left[r, z-z_{i}(N), a_{i}(N)\right],
\end{aligned}
$$

где функция $b_{z}\left[r, z-z_{i}(N), a_{i}(N)\right]-$ продольный компонент вектора индукции магнитного поля, создаваемого тонким кольцом радиуса $a_{i}(N)$ с единичным током в точках пространства, задаваемых в цилиндрической системе координатами $r$ и $z$. Функция $b_{z}$ определяется следующим соотношением [3]:

$$
\begin{aligned}
b_{z}(r, z, a)= & \frac{\mu_{0}}{2 \pi}\left\{E[w(r, z, a)] \frac{a^{2}-r^{2}-z^{2}}{(a-r)^{2}+z^{2}}\right. \\
& +K[w(r, z, f)]\} \frac{1}{\sqrt{(a+r)^{2}+z^{2}}},
\end{aligned}
$$

где

$$
w(r, z, a)=\frac{4 a r}{(a+r)^{2}+z^{2}},
$$

$\mu_{0}$ - магнитная постоянная, $a-$ радиус кольца, $K(w)$ и $E(w)$ - полные эллиптические интегралы. Для выбранной геометрии спирали и различных отклонений от оси $\left(0<r<4 \cdot 10^{-3} \mathrm{~m}\right)$ получены расчетные зависимости пространственных амплитуд продольного магнитного поля

$$
B_{0 z}(r, z, N)=\sum_{i=1}^{N} b_{z}\left[r, z-z_{i}(N), a_{i}(N)\right]
$$

В качестве иллюстрации на рис. 2 представлен один из вариантов такого расчета. Анализ результатов компьютерного моделирования показал, что для небольших $N$ эффективное ускорение происходит в самом начале спиральной линии на длине $\sim R_{\min }$, причем преимущественно для частиц из периферийной плазмы.

Для корректного учета влияния параметров линии на работу ускорителя в целом нужно знать ее индуктивность. В основу алгоритма расчета индуктивности $L(N)$ спиральной линии легло известное выражение

$$
\frac{d \Phi}{d t}=L \frac{d I}{d t}
$$

в котором магнитный поток определяется формулой

$$
\Phi(t)=2 \pi I(t) \sum_{i=1}^{N} \int_{0}^{a_{i}(N)} r B_{0 z}\left(r, z_{i}, N\right) d r .
$$

Из этих соотношений следует выражение для расчета индуктивности

$$
L(N)=2 \pi \sum_{i=1}^{N} \int_{0}^{a_{i}(N)} r B_{0 z}\left(r, z_{i}, N\right) d r .
$$

В диапазоне $1 \leq N \leq 8$ индуктивность спиральной линии изменяется в довольно широких пределах от $0.05 \cdot 10^{-6}$ до $2 \cdot 10^{-6} \mathrm{H}$, и ее необходимо учитывать в расчетной формуле для импульса тока в разрядном контуре

$$
I(t)=I_{0} \exp (-\alpha t) \sin (\omega t+\beta),
$$

где $I_{0}=U /\left\{\omega_{0}\left[L(N)+L_{G}\right] \cos \beta\right\}, \quad \alpha=R / 2\left[L(N)+L_{G}\right]$, $\beta=\operatorname{arctg}(\alpha / \omega), \omega_{0}=\left\{\left[L(N)+L_{G}\right] C\right\}^{-0.5}, \omega=\left(\omega_{0}^{2}-\beta^{2}\right)^{0.5}$, 
$U$ - напряжение, запасаемое в емкостном накопителе энергии зарядки, $C$ и $L_{G}-$ соответственно емкость и индуктивность накопителя энергии импульсного генератора тока, $R$ - активное сопротивление разрядной цепи.

Для анализа процесса ускорения ЛП в магнитном поле спиральной линии предложена математическая модель, основанная на взаимодействии магнитного момента плазменного сгустка с градиентом спада продольного магнитного поля. В процессе нарастания магнитного поля в ЛП возникает азимутальный электрический ток с плотностью

$$
j(r, z, t) \approx \sigma[\theta(t)] \frac{r}{2} \frac{d I}{d t} \sum_{i=1}^{N} B_{z}\left(r, z-z_{i}, a_{i}\right),
$$

где $\sigma$ - проводимость плазмы с температурой $\theta$. Ему соответствует магнитный момент плазменного сгустка

$$
\mu\left(t, z_{c}, r_{c}\right) \approx \pi \int_{0}^{2\left(r_{0}+z_{c}\right)} d z \int_{0}^{r_{c}} d r r^{2} j(r, z, t),
$$

где $z_{c}$ и $r_{c}$ - текущие продольная координата центра тяжести плазменного сгустка и среднее радиальное отклонение фронта ЛП от оси. На плазменный сгусток будет действовать сила [4]:

$$
F\left(t, z_{c}, r_{c}\right) \approx-\left.\mu\left(t, z_{c}, r_{c}\right) \frac{\partial}{\partial z} B_{z}(0, z, t)\right|_{z=z_{c}} .
$$

Анализ экспериментальных данных по кинематике разлета ЛП [5] показал, что для оценочных расчетов вполне приемлема модель, в которой разлет плазмы можно представить в виде расширяющегося сферического плазменного облака, центр которого движется в направлении, перпендикулярном поверхности лазерной мишени, со скоростью распространения плазменного фронта $V_{f}$. Поле скоростей при этом будет определяться следующим выражением:

$$
\mathbf{V}(\mathbf{r}, t)=\mathbf{e}_{r} \frac{r V_{f}}{V_{f} t+r_{0}}+\mathbf{e}_{z} V_{f}\left(\frac{z}{V_{f} t+r_{0}}+1\right) .
$$

При наличии продольного магнитного поля индикатриса плазменного потока сужается и поле скоростей будет определяться выражением

$$
\mathbf{V}(\mathbf{r}, t)=\mathbf{e}_{r} \frac{r V_{\perp}(t)}{V_{\perp}(t) t+r_{0}}+\mathbf{e}_{z} V_{f}\left(\frac{z}{V_{f} t+r_{0}}+1\right) .
$$

где, согласно [6], $V_{\perp}(t) \approx 0.7 V_{f}\left[1+1.9 B_{z}^{1.2}(t)\right]^{-1.2}$.

Движение центра тяжести ЛП определяется следующими уравнениями:

$$
\Delta m \frac{d V_{c}}{d t} \approx F\left(t, z_{c}, r_{c}\right), \quad \frac{d z_{c}}{d t}=V_{c}, \quad \frac{d r_{c}}{d t} \approx V_{\perp}(t),
$$

где $\Delta m \approx 2 \cdot 10^{-12} \mathrm{~kg}$ - масса плазменного сгустка на стадии закалки его ионизационного состояния с начальными условиями $V_{c}(0)=V_{0}, z_{c}(0)=r_{0}, r_{c}(0)=r_{0}$, полученными из выражения для поля скоростей, представленного выше. При этом временна́я зависимость средней

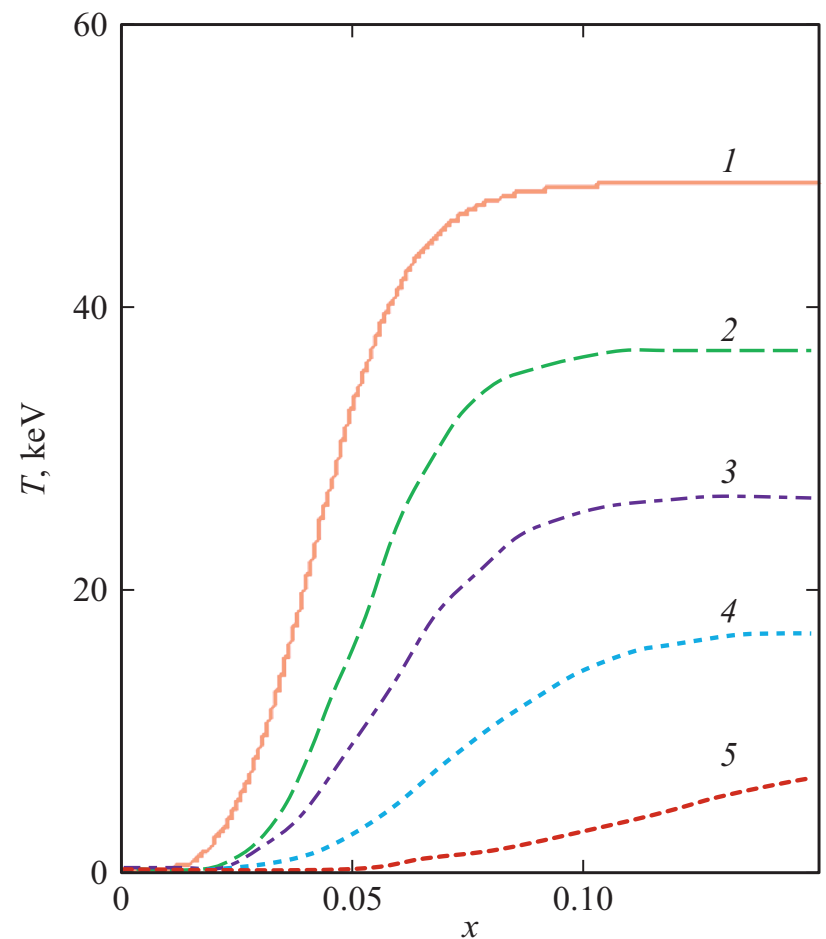

Рис. 3. Семейство расчетных зависимостей набора энергии дейтрона, усредненной по плазменному сгустку, от безразмерного времени $x=\omega t$, построенное для различных значений амплитуды возбуждаемого в спирали тока. $I_{0}, \mathrm{kA}: 1-50$, $2-40,3-30,4-20,5-10$.

энергии дейтрона в сгустке определяется формулой

$$
T=\left(10^{-3} M / 2 e\right) V_{c}^{2}[\mathrm{keV}] .
$$

На рис. 3 представлены расчетные зависимости энергии $T$ от безразмерного времени $x=\omega t$, полученные для задержки $\tau=100 \mathrm{~ns}$ между импульсами лазерного излучения и тока, генерируемого в спирали с числом витков $N=5$. Средняя температура плазмы оценивалась на уровне $3 \mathrm{eV}$ на основании пирометрических измерений с использованием коаксиального фотоэлемента. Максимальная средняя скорость дейтронов, полученная при компьютерных расчетах, составила величину около $2 \cdot 10^{6} \mathrm{~m} / \mathrm{s}$. Это значение слабо менялось в диапазоне изменения энергии лазерного импульса от 0.1 до $0.5 \mathrm{~J}$ при длительности лазерного импульса $10^{-8} \mathrm{~s}$.

В настоящее время проведены первые эксперименты по ускорению лазерной плазмы спиралью для геометрических параметров и энергетики, соответствующих описанному выше компьютерному эксперименту. С помощью временно́го анализа удалось оценить максимальную скорость ускоренных дейтронов, соответствующую переднему фронту ЛП, на уровне около $5 \cdot 10^{6} \mathrm{~m} / \mathrm{s}$. На основании этих предварительных данных можно, опираясь на представленную выше формулу для поля скоростей, говорить об адекватности предложенной модели ускорения ЛП импульсным магнитным полем 
спиральной линии и возможности ее использования для прогнозирования результатов дальнейших экспериментов и проектирования систем для генерации нейтронов.

Работа выполнена при финансовой поддержке Министерства науки и образования РФ по соглашению № 14.575.21.0169 (RFMEFI57517X0169).

\section{Список литературы}

[1] Vovchenco E.D., Isaev A.A., Kozlovskij K.I., Shikanov A.E. // J. Phys.: Conf. Ser. 2017. V. 941. P. 012016.

[2] Миллер Р. Введение в физику сильноточных пучков заряженных частиц. М.: Мир, 1984. 432 с.

[3] Ландау Л.Д., Лифиии, Е.M. Электродинамика сплошных сред. М.: Наука, 1982. 624 с.

[4] Тамм И.Е. Основы теории электричества. М.: ГИТТЛ, 1954. $620 \mathrm{c}$.

[5] Цыбин А.С. Динамика лазерной плазмы в сильноточных импульсных диодных системах // Радиационная плазмодинамика. М.: Энергоатомиздат, 1991. Т. 1. С. 437-462.

[6] Диденко А.Н., Шиканов А.Е., Козловский К.И., Шатохин В.Л., Пономарев Д.Д. // Физика плазмы. 2014. Т. 40. № 11. C. $1025-1034$. 